Journal of

\section{Synchrotron}

Radiation

ISSN 0909-0495

Received 12 June 2013

Accepted 7 August 2013

\title{
Cryo-electron tomography: moving towards revealing the viral life cycle of Rice dwarf virus
}

\author{
Naoyuki Miyazaki, ${ }^{\text {a,b* }}$ Fusamichi Akita, ${ }^{\text {c,d }}$ Atsushi Nakagawa, ${ }^{b}$ Kazuyoshi Murata, ${ }^{a}$ \\ Toshihiro Omura ${ }^{c}$ and Kenji Iwasaki ${ }^{b *}$
}

\begin{abstract}
${ }^{a}$ National Institute for Physiological Sciences, 38 Nishigonaka, Myodaiji, Okazaki, Aichi 444-8585, Japan, 'bnstitute for Protein Research, 3-2 Yamadaoka, Suita, Osaka 565-0871, Japan, 'National Agricultural Research Center, 3-1-1 Kannondai, Tsukuba, Ibaraki 305-8666, Japan, and dDivision of Bioscience, Graduate School of Natural Science and Technology, Faculty of Science, Okayama University, Okayama 700-8530, Japan. E-mail: naomiya@nips.ac.jp, ikenji@protein.osaka-u.ac.jp
\end{abstract}

\begin{abstract}
It is well known that viruses utilize the host cellular systems for their infection and replication processes. However, the molecular mechanisms underlying these processes are poorly understood for most viruses. To understand these molecular mechanisms, it is essential to observe the viral and virus-related structures and analyse their molecular interactions within a cellular context. Cryo-electron microscopy and tomography offer the potential to observe macromolecular structures and to analyse their molecular interactions within the cell. Here, using cryo-electron microscopy and tomography, the structures of Rice dwarf virus are reported within fully hydrated insect vector cells grown on electron microscopy grids towards revealing the viral infection and replication mechanisms.
\end{abstract}

Keywords: Rice dwarf virus; Phytoreovirus; virus structure; cryo-electron tomography; cryo-electron microscopy.

\section{Introduction}

Rice dwarf virus (RDV), a member of the family Reoviridae, is an icosahedral double-layered particle of approximately $70 \mathrm{~nm}$ in diameter (Nakagawa et al., 2003; Miyazaki et al., 2005), and the agent of rice dwarf disease causing economic damage in many Asian countries. RDV has a 12-segmented dsRNA genome encoding 12 viral proteins. The RDV particle is composed of seven structural proteins ( $\mathrm{P} 1, \mathrm{P} 2, \mathrm{P} 3, \mathrm{P} 5, \mathrm{P} 7, \mathrm{P} 8$ and $\mathrm{P} 9$ ). The P3 structural proteins form the inner capsid shell which encapsidates the viral genome and the P1, P5 and P7 proteins are required for transcription (Hagiwara et al., 2003, 2004; Miyazaki et al., 2010b). The inner capsid shell is surrounded by the outer capsid shell, which consists of P2, P8 and P9 proteins (Omura \& Yan, 1999). Five kinds of nonstructural proteins (Pns4, Pns6, Pns10, Pns11 and Pns12) are related to viral replication within the host cell, including intracellular trafficking (Wei et al., 2006b), synthesis of viral genome and proteins, assembly of progeny viruses (Wei et al., 2006c; Shimizu et al., 2009), viral release from infected cells (Wei et al., 2008, 2009; Miyazaki et al., 2010a.), and intercellular transport (Wei et al., 2006a). However, the structural details underlying these controlled events are poorly understood for RDV and most other viruses. Cryo-electron microscopy (cryo-EM) and tomography (cryo-ET) can be used to observe hydrated cells in a close-to-native state at molecular resolution, which allows analysis of molecular interactions within a cell (Robinson et al., 2007). Here, we show one of the workable strategies using cryo-EM/ET to study viral infection and replication mechanisms.

\section{Materials and methods}

2.1. Cell culture and virus infection on EM grids, and rapid freezing

NC24 cells, originally established from embryonic fragments dissected from the eggs of the leafhopper Nephotettix cincticeps (the insect vector of RDV), were maintained in monolayer culture at $298 \mathrm{~K}$ in growth medium that was prepared as previously described (Kimura, 1986). To examine the virus-infected NC24 cells by cryo-EM/ET, the NC24 cells were grown on Quantifoil holey carbon supported gold EM grids. The cells were further cultivated for three to five days after inoculation with RDV at viral dilutions required to reach 100\% infection (Kimura, 1986; Wei et al., 2006a), and were plunged frozen in liquid ethane and embedded in vitreous ice using a Vitrobot (FEI, The Netherland).

\subsection{Cryo-EM/ET observations}

The EM grids were stored in liquid nitrogen and transferred into a cryo-electron microscope (Titan-Krios; FEI, The Netherlands). The Titan-Krios was operated at $200 \mathrm{kV}$, and incorporated a field emission gun, a liquid-nitrogen stage and 
a Gatan 4096 × 4096 CCD camera (Model UltraScan 4000; Gatan, USA). The cryo-EM images were recorded under lowdose conditions $\left(\sim 20 \mathrm{e}^{-} \AA^{-2}\right)$. To determine three-dimensional structure by cryo-ET, a tilt-series was collected from $-60^{\circ}$ to $+60^{\circ}$ with an angular increment of $2^{\circ}$ on the Gatan $4096 \times 4096$ CCD camera in the Titan-Krios. The total electron dose in the acquisition of the tilt series was kept under $60 \mathrm{e}^{-} \AA^{-2}$. The tilt series was aligned using $10 \mathrm{~nm}$ colloidal gold particles as fiducial markers. Three-dimensional reconstructions were calculated using the software IMOD (Kremer et al., 1996).

\section{Results and discussion}

\subsection{Cryo-EM/ET observations of the cellular structures under close-to-native conditions}

Electron microscopy (EM) has long been used for highresolution analysis of cellular and viral structures. Conventional EM sample preparation methods consist of chemical fixation, dehydration and resin embedding of biological specimens. The resin-embedded specimens are then cut into ultra-thin sections, and examined by a transmission electron microscope after staining with electron-dense heavy metals. However, the conventional EM methods cause structural rearrangements, aggregation of cellular contents, and loss of unfixed materials during the sample preparation process, which impedes high-resolution structural analysis. Compared with the conventional method, the advantage of cryo-EM/ET is that the specimens are observed in fully hydrated conditions, which avoids the chemical fixation, dehydration, resin embedding and staining processes. Thus, it limits structural rearrangements or aggregation during the sample preparation as much as possible, which allows us to analyse the fine cellular structures in a close-tonative state. In this study, we used cryo$\mathrm{EM} / \mathrm{ET}$ to analyse the structures of the RDV particles within the insect vector (NC24) cells. The NC24 cells adhered to the carbon supported film on the EM grid, and were maintained on the EM grid for the required time for RDV to replicate (Fig. 1a). When the cells were examined by cryo-EM, the cellular structures at the periphery were clearly visible (Fig. 1b). Dark spherical densities at the edge of the cells, as seen in Fig. 1(b), correspond to the cellular vesicles or organelles. Moreover, the cells were well vitrified without any ice crystal formation in the specimen, and the fine cellular structures were well preserved. Examined at higher magnification, we were able to observe the fine cellular structures in detail. For

Figure 1 region. Bar: $400 \mathrm{~nm}$. example, in Fig. 1(c), the fine membrane structures of mitochondria are clearly visualized by cryo-EM.

\subsection{Cryo-EM/ET observations of the virus structures within the host cells}

Viruses must be released from infected cells for successful spreading. In the case of RDV, viral egress from the insect vector cells utilizes multiple pathways without cell lysis. In one of these pathways, multivesicular compartments are involved in the release of RDV particles from the cells (Wei et al., 2008, 2009; Miyazaki et al., 2010a). The proposed RDV release pathway is that newly synthesized progeny viruses at the viroplasm (Wei et al., 2006c) are engulfed by multivesicular compartments that move to the periphery of cells, where they fuse with the plasma membrane to facilitate release of viral particles (Wei et al., 2008, 2009). In this study, cryo-EM/ET observations clearly visualized the RDV particles within multi-vesicular bodies at the edge of the cell, which appear to be in a state prior to egress from the infected NC24 cell (Fig. 2). This result demonstrates the success of our approach using cryo-EM/ET techniques to observe the viral and virus-related structures within cells and to analyse the viral life cycle around the edge of the infected cells. On the other hand, the central part of the cell was very dark, and the cellular and viral structures were invisible, due to the sample thickness being too large for the electron beam to penetrate (Fig. 1b). Thus, the limited thickness range of $0.5-1 \mu \mathrm{m}$ that is accessible with standard accelerating voltage electron microscopes (under $500 \mathrm{kV}$ ) restricts observations to only the extreme edge
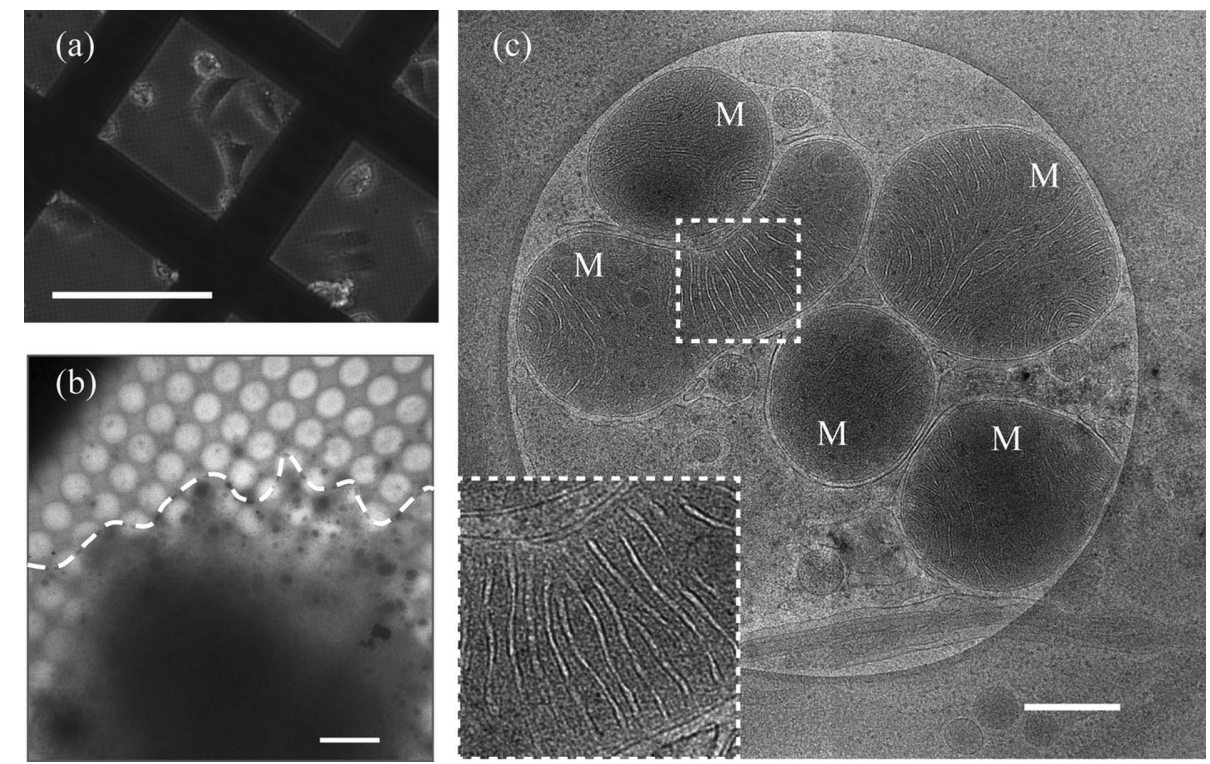

Observations of NC24 cells (the insect vector cells of RDV) cultivated on EM grids. (a) A light microscopy image of RDV-infected NC24 cells grown on an EM grid. Bar: $100 \mu \mathrm{m}$. (b) Lowmagnification cryo-EM image of the NC24 cell cultured on the EM grid. The cell is outlined with a white dashed line. The cell periphery was thin enough to allow observation of the cellular structures, while the central part of the cell was too thick for imaging and appeared as a dark region. Bar: $4 \mu \mathrm{m}$. (c) High-magnification cryo-EM image of the NC24 cells cultivated on the EM grid. The fine membrane structures of mitochondria (M) were clearly visible. (Inset) Enlarged view of the boxed 


\section{diffraction structural biology}
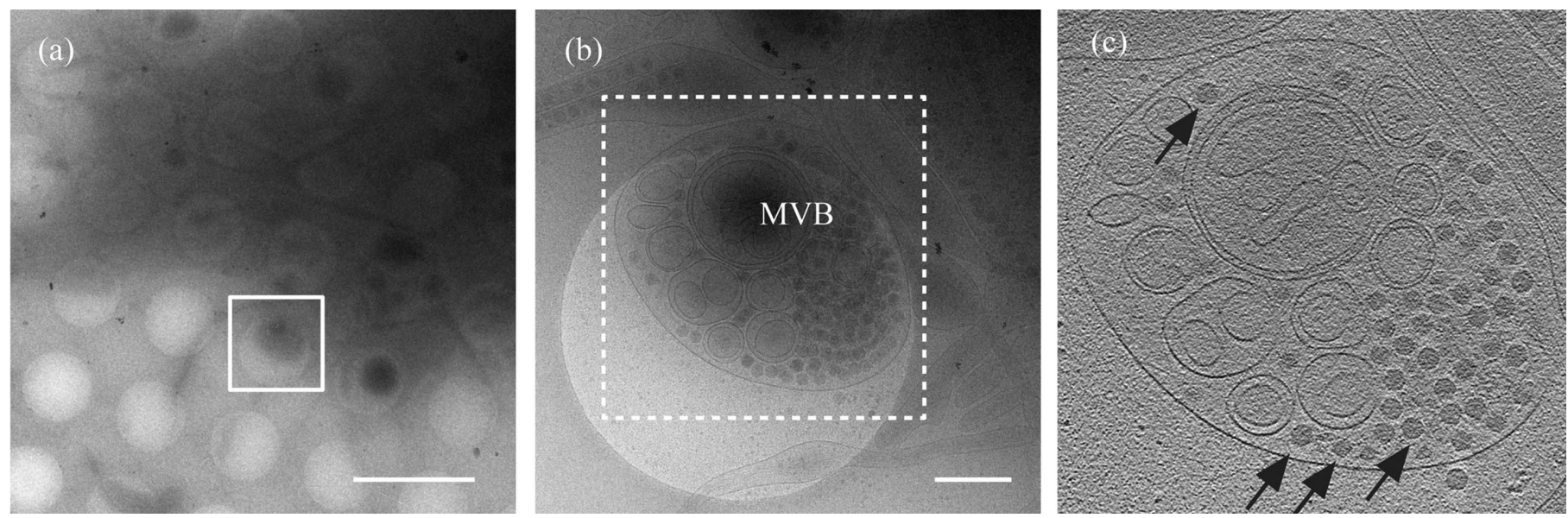

Figure 2

Cryo-EM/ET observations of the RDV structures within the insect vector cell. (a) Low-magnification cryo-EM image of the RDV-infected NC24 cell cultured on the EM grid. Bar: $2 \mu \mathrm{m}$. (b) High-magnification cryo-EM image of the boxed region in $(a)$. Bar: $400 \mathrm{~nm}$. $(c)$ A slice through the reconstructed tomographic volume obtained from the area highlighted in $(b)$. Black arrows indicate the RDV particles within the multivesicular body (MVB).

portions of the cell. In order to study the complete viral life cycle, the whole cell body must be examined by cryo-EM/ET. This restriction may be overcome by using a high-voltage electron microscope, because the highly accelerated electrons (over $500 \mathrm{keV}$ ) can penetrate thicker specimens. Alternatively, processing a sample into thin vitreous films using CEMOVIS (cryo-electron microscopy of vitreous sections; AlAmoudi et al., 2005; Dubochet \& Blanc, 2001) or cryo-FIB (cryo-focused ion beam; Wang et al., 2012) may allow observation of thicker samples. Cryo-EM/ET observations of such thick specimens are now underway.

This work was supported by CREST; the Japan Science and Technology Agency; a Grant-in-Aid for Scientific Research on Priority Areas (Structures of Biological Macromolecular Assemblies) and KAKENHI grant No. 25251009 from the Ministry of Education, Culture, Sports, Science and Technology of Japan; and the Program for Promotion of Basic Research Activities for Innovative Biosciences of the Biooriented Technology Research Advancement Institution (BRAIN), Japan.

\section{References}

Al-Amoudi, A., Chang, J. J., Leforestier, A., McDowall, A., Salamin, L. M., Norlen, L. P., Richter, K., Blanc, N. S., Studer, D. \& Dubochet, J. (2005). EMBO J. 23, 3583-3588.

Dubochet, J. \& Sartori Blanc, N. (2001). Micron, 32, 91-99.

Hagiwara, K., Higashi, T., Miyazaki, N., Naitow, H., Cheng, R. H., Nakagawa, A., Mizuno, H., Tsukihara, T. \& Omura, T. (2004). J. Virol. 78, 3145-3148.
Hagiwara, K., Higashi, T., Namba, K., Uehara-Ichiki, T. \& Toshihiro, O. (2003). J. Gen. Virol. 84, 1-4.

Kimura, I. (1986). J. Gen. Virol. 71, 1861-1863.

Kremer, J. R., Mastronarde, D. N. \& McIntosh, J. R. (1996). J. Struct. Biol. 116, 71-76.

Miyazaki, N., Hagiwara, K., Naitow, H., Higashi, T., Cheng, R. H., Tsukihara, T., Nakagawa, A. \& Omura, T. (2005). J. Mol. Biol. 345, 229-237.

Miyazaki, N., Hagiwara, K., Wei, T., Chen, H., Nakagawa, A., Xing, L., Cheng, R. H. \& Omura, T. (2010a). J. Gen. Virol. 91, 28572861.

Miyazaki, N., Wu, B., Hagiwara, K., Wang, C.-Y., Xing, L., Hammar, L., Higashiura, A., Tsukihara, T., Nakagawa, A., Omura, T. \& Cheng, R. H. (2010b). J. Biochem. 147, 843-850.

Nakagawa, A., Miyazaki, N., Taka, J., Naitow, H., Ogawa, A., Fujimoto, Z., Mizuno, H., Higashi, T., Watanabe, Y., Omura, T., Cheng, R. H. \& Tsukihara, T. (2003). Structure, 11, 1227-1238.

Omura, T. \& Yan, J. (1999). Adv. Virus Res. 54, 15-43.

Robinson, C. V., Sali, A. \& Baumeister, W. (2007). Nature (London), 450, 973-982.

Shimizu, T., Yoshii, M., Wei, T., Hirochika, H. \& Omura, T. (2009). Plant Biotechnol. J. 7, 24-32.

Wang, K., Strunk, K., Zhao, G., Gray, J. L. \& Zhang, P. (2012). J. Struct. Biol. 180, 318-326.

Wei, T., Hibino, H. \& Omura, T. (2008). J. Gen. Virol. 89, 29152920.

Wei, T., Hibino, H. \& Omura, T. (2009). Commun. Integr. Biol. 2, 324 326.

Wei, T., Kikuchi, A., Moriyasu, Y., Suzuki, N., Shimizu, T., Hagiwara, K., Chen, H., Takahashi, M., Ichiki-Uehara, T. \& Omura, T. (2006a). J. Virol. 80, 8593-8602.

Wei, T., Kikuchi, A., Suzuki, N., Shimizu, T., Hagiwara, K., Chen, H. \& Omura, T. (2006b). Arch. Virol. 151, 1701-1712.

Wei, T., Shimizu, T., Hagiwara, K., Kikuchi, A., Moriyasu, Y., Suzuki, N., Chen, H. \& Omura, T. (2006c). J. Gen. Virol. 87, 429-438. 